PROCEEDINGS OF THE

AMERICAN MATHEMATICAL SOCIETY

Volume 131, Number 6, Pages 1901-1906

S 0002-9939(03)06879-5

Article electronically published on January 8, 2003

\title{
RANDOM MENSHOV SPECTRA
}

\author{
GADY KOZMA AND ALEXANDER OLEVSKIǏ \\ (Communicated by Andreas Seeger)
}

\begin{abstract}
We show that the spectra $\Lambda$ of frequencies $\lambda$ obtained by random perturbations of the integers allows one to represent any measurable function $f$ on $\mathbb{R}$ by an almost everywhere converging sum of harmonics:

$$
f=\sum_{\Lambda} c_{\lambda} e^{i \lambda t}
$$
\end{abstract}

\section{INTRODUCTION}

This paper concerns the representation of functions by series of exponentials which converge almost everywhere (a.e.). According to Menshov's theorem (1941, see [1]) every $2 \pi$-periodic measurable function $f$ admits a representation as

$$
f(t)=\sum_{k \in \mathbb{Z}} c(k) e^{i k t} \quad \text { a.e. }
$$

Among the many generalizations and analogs of this fundamental result, there exists a version for the non-periodic case: Davtjan [2] proved that the corresponding representation on $\mathbb{R}$ can be obtained if instead of the sum over integers one considers a "trigonometric integral" which involves all real frequencies.

In our paper [4] it was shown that most of the frequencies are redundant. Namely, by appropriate small perturbations of the integers we constructed a spectrum of frequencies $\Lambda=\{\lambda(k), k \in \mathbb{Z}\}$ such that any $f \in L^{0}(\mathbb{R})$ (that is, a measurable function on $\mathbb{R})$ can be decomposed as

$$
f(t)=\sum_{k \in \mathbb{Z}} c(k) e^{i \lambda(k) t} \quad \text { a.e. }
$$

The aim of this note is to show that this is not an exceptional feature of the constructed spectrum. In fact, by choosing the perturbations randomly one gets such a property with probability 1 .

Analogously to the periodic case (see [5]) we introduce the following

Definition. A sequence $\Lambda=\{\ldots<\lambda(k)<\ldots<\lambda(-1)<\lambda(0)<\lambda(1)<\ldots\}$ is called a Menshov spectrum for $\mathbb{R}$ if for any $f \in L^{0}(\mathbb{R})$ there are coefficients $\{c(k)\}$ such that the decomposition (22) holds (convergence in (2) is understood in the sense of the limit of symmetric partial sums, i.e. $\lim _{x \rightarrow \infty} \sum_{|\lambda|<x}$ ).

Received by the editors February 8, 2002.

2000 Mathematics Subject Classification. Primary 42A63, 42A61, 42 A55.

Key words and phrases. Random spectra, representation of functions by trigonometric series. Research supported in part by the Israel Science Foundation.

(C)2003 American Mathematical Society 
Theorem. Let $r(n)$ be independent variables uniformly distributed on the segment $\left[-\frac{1}{2}, \frac{1}{2}\right]$. Then the sequence

$$
\lambda(n)=n+r(n), \quad n \in \mathbb{Z},
$$

is almost surely a Menshov spectrum for $\mathbb{R}$.

The proof is based on the technique used in our recent paper [5]. We refer the reader to this paper for some historical comments and additional references.

\section{Preliminaries}

By a trigonometric polynomial $P$ we mean a finite linear combination of exponentials with real (not necessarily integer) frequencies $\ldots<\lambda(-1)<\lambda(0)<\ldots$. We call the set of $\lambda$ 's involved, the spectrum of $P$ and denote it by $\operatorname{spec} P$. The corresponding coefficients are denoted as $\widehat{P}(\lambda)$, so

$$
P=\sum_{\operatorname{spec} P} \widehat{P}(\lambda) e^{i \lambda x}, \quad x \in \mathbb{R} .
$$

We denote

$$
\operatorname{deg} P=\max _{\operatorname{spec} P}|\lambda|
$$

As usual

$$
\|\widehat{P}\|_{1}:=\sum|\widehat{P}(\lambda)|, \quad\|\widehat{P}\|_{\infty}:=\max |\widehat{P}(\lambda)| .
$$

Let $P^{*}$ be the (non-symmetric) majorant of partial sums:

$$
P^{*}(x):=\max _{a<b}\left|\sum_{\operatorname{spec} P \cap[a, b]} \widehat{P}(\lambda) e^{i \lambda x}\right| .
$$

For a given $P$ and $l \in \mathbb{Z}^{+}$we denote by $P_{[l]}$ the "contracted" polynomial:

$$
P_{[l]}(x)=P(l x) .
$$

The following "special products" are used:

$$
H=Q_{[l]} P .
$$

If $\operatorname{spec} Q \subset \mathbb{Z}$ and $l>2 \operatorname{deg} P$, then this product has a simple structure which provides the following estimate (compare with (10) in [5]):

$$
H^{*}(x) \leq|P(x)| \cdot\left\|Q^{*}\right\|_{L^{\infty}(-\pi, \pi)}+2 P^{*}(x) \cdot\|\widehat{Q}\|_{\infty} .
$$

We will use the following

Lemma 1 (see [5], Lemma 2.1). Given any $\epsilon>0, \delta>0$, there exists a trigonometric polynomial $P=P_{\epsilon, \delta}$ with integer spectrum such that

(i) $\widehat{P}(0)=0,\|\widehat{P}\|_{\infty}<\delta$;

(ii) $\mathbf{m}\{x \in[-\pi, \pi]:|P(x)-1|>\delta\}<\epsilon$;

(iii) $\left\|P^{*}\right\|_{\infty}<C \epsilon^{-1}$. 


\section{Proof of THE THEOREM}

3.1. The result is an easy consequence of the following (nonstochastic)

Proposition. Let $\Lambda=\{\lambda(n)\}, \lambda(n)=n+r(n), n \in \mathbb{Z}$, and suppose that for every $k \in \mathbb{Z}^{+}$there exists a number $l=l(k)$ in $\mathbb{Z}^{+}$such that

$$
\left|r(s l+q)-2^{-|q|+1}\right|<\frac{1}{k^{2}}, \quad 0<|s|<k,|q|<k .
$$

Then $\Lambda$ is a Menshov spectrum for $\mathbb{R}$.

To deduce the theorem from the proposition it is enough to mention that if we fix $k$ and run $l$ over a sufficiently fast increasing sequence $\left\{l_{j}\right\}$, then the events $B_{j}$ that the inequalities above are fulfilled for $l=l_{j}$ are mutually independent and each has a positive probability $p(k)$ which does not depend on $j$. So for a random spectrum $H$ the condition of the proposition is true almost surely.

3.2. Now we pass to the proof of the proposition. Denote $I(k):=\{s l(k)+q$ : $0<|s|<k,|q|<k\}$ and $M(k)=k l(k)+k$. Clearly (passing to a subsequence if necessary) we may suppose that

$$
I(k+1) \cap[-M(k), M(k)]=\emptyset .
$$

Let $f \in L^{0}(\mathbb{R})$ be given. We shall define by induction an increasing sequence $\left\{k_{j}\right\}$ and "blocks" of coefficients $\{c(n)\}, n \in I\left(k_{j}\right)$; all other coefficients of the expansion (2) will be zero. We denote:

$$
\begin{aligned}
A_{j} & :=\sum_{I\left(k_{j}\right)} c(n) \exp (i \lambda(n) x), \\
S_{N} & :=\sum_{j \leq N} A_{j} \quad N \in \mathbb{Z}^{+}, S_{0}:=0 .
\end{aligned}
$$

Fix $N$ and suppose that the polynomials $A_{j}$ are already defined for $j<N$. Let us describe the $N$ 'th step of the induction. Set

$$
R_{N}:=f-S_{N-1} .
$$

We need the following result proved in [3]:

if $0<|r(q)|=o(1)$, then the system of exponentials $\exp i(q+r(q)), q \in \mathbb{Z}$, is complete in $L^{0}(\mathbb{R})$, that is, the set of linear combinations is dense with respect to convergence a.e.

Using this we find a polynomial

$$
F_{N}(x)=\sum a_{q} \exp i\left(q+2^{-|q|+1}\right) x
$$

so that

$$
\mathbf{m}\left\{x \in[-N \pi, N \pi]:\left|F_{N}(x)-R_{N}(x)\right|>\frac{1}{N^{4}}\right\}<\frac{1}{N^{2}} .
$$

Next we use Lemma 1 with

$$
\delta=\delta_{N}=\frac{1}{N^{4}\left\|\widehat{F_{N}}\right\|_{1}}, \quad \epsilon=\frac{1}{N^{3}}
$$

and find the corresponding polynomial $Q_{N}$. Fix a number $k_{N}$ large enough:

$$
k_{N}>k_{N-1}, 3 \operatorname{deg} F_{N}, \frac{\left\|\widehat{Q_{N}}\right\|_{1}}{\delta_{N}}
$$


and set

$$
H_{N}:=F_{N} \cdot\left(Q_{N}\right)_{\left[l\left(k_{N}\right)\right]} .
$$

One can easily see that

$$
\operatorname{spec} H \subset\left\{s l\left(k_{N}\right)+q+2^{-|q|+1}: 0<|s|<k,|q|<k\right\},
$$

so we can write

$$
H_{N}=\sum_{\substack{0<|s|<k_{N} \\|q|<k_{N}}} b(N, s, q) \exp i\left(s l\left(k_{N}\right)+q+2^{-|q|+1}\right) x .
$$

Finally we set:

$$
A_{N}:=\sum_{\substack{0<|s|<k_{N} \\|q|<k_{N}}} b(N, s, q) \exp i \lambda\left(s l\left(k_{N}\right)+q\right) x \equiv \sum_{I\left(k_{N}\right)} c(n) \exp i \lambda(n) x .
$$

3.3. Now we show that

$$
S_{N} \rightarrow f \quad \text { a.e. }
$$

For this first we get from (ii) of Lemma 1

$$
\begin{aligned}
\mathbf{m}(\{x & \left.\left.\in[-N \pi, N \pi]:\left|H_{N}(x)-F_{N}(x)\right|>\frac{1}{N^{4}}\right\}\right) \\
& \leq N \cdot \mathbf{m}\left(\left\{x \in[-\pi, \pi]:\left|Q_{N}-1\right| \geq \delta_{N}\right\}\right)=O\left(N^{-2}\right) .
\end{aligned}
$$

Further, (9), (8) and (7) imply:

$$
\left\|\widehat{H_{N}}\right\|_{1}=\left\|\widehat{F_{N}}\right\|_{1} \cdot\left\|\widehat{Q_{N}}\right\|_{1}<\left\|\widehat{F_{N}}\right\|_{1} k_{N} \delta_{N}=\frac{k_{N}}{N^{4}},
$$

so we can estimate, using (10), (11), (5), (14), (3) and (8):

$$
\begin{aligned}
\| A_{N}- & H_{N} \|_{L^{\infty}(-\pi N, \pi N)} \\
& \leq\left\|\widehat{H_{N}}\right\|_{1} \cdot \max _{\substack{0<|s|<k_{N} \\
|q|<k_{N}}}\left\|\exp i\left(r\left(\operatorname{sl}\left(k_{N}\right)+q\right)-2^{-|q|+1}\right) x-1\right\|_{\infty} \\
& <\frac{k_{N}}{N^{4}} \cdot \frac{1}{k_{N}^{2}} \cdot \pi N=O\left(N^{-4}\right) .
\end{aligned}
$$

Finally, we have from (6), (13) and (15):

$$
\mathbf{m}\left\{x \in[-N \pi, N \pi]:\left|A_{N}(x)-R_{N}(x)\right|>\frac{C}{N^{4}}\right\}=O\left(N^{-2}\right),
$$

so

$$
R_{N+1}=R_{N}-A_{N}=O\left(N^{-4}\right) \quad \text { a.e. }
$$

and (12) follows. 


\subsection{At last:}

$$
A_{N}^{*} \rightarrow 0 \quad \text { a.e. }
$$

Indeed, estimating as in (15) we see:

$$
A_{N}^{*}<H_{N}^{*}+O\left(N^{-4}\right) \text { a.e. }
$$

Since $l\left(k_{N}\right)>k_{N}>3 \operatorname{deg} F_{N}$ we can use (4) and get:

$$
H_{N}^{*}(x)<\left|F_{N}(x)\right| \cdot\left\|Q_{N}^{*}\right\|_{L^{\infty}(-\pi, \pi)}+2\|\widehat{Q}\|_{\infty} \cdot\|\widehat{F}\|_{1} .
$$

The first term on the right hand side is $O\left(N^{-1}\right)$ a.e. due to (66), (16) and (iii) The last term is $O\left(N^{-4}\right)$ because of (i) and (7). Clearly (12) and (17) imply the decomposition (2) and this completes the proof.

\section{REMARKS}

4.1. One can see that the result holds for $r(n)$ uniformly distributed on any fixed neighbourhood of zero. Moreover, it holds true for $r(n)$ uniformly distributed on $\left[-d_{n}, d_{n}\right]$ if $d_{n}$ decrease slowly enough. This allows one to cover in full generality the result from 4 where a Menshov spectrum $\{n+o(1)\}$ was constructed. But $d_{n}$ really must decay slowly. In contrast to the completeness property which occurs for any (nonzero) perturbation $r(n)=o(1)$ (the result from [3] used above), the following simple observation is true:

If $\lambda(n)=n+O\left(n^{-\alpha}\right), \alpha>0$, then it is not a Menshov spectrum.

Indeed, if $\Delta f:=f(x)-f(x-2 \pi)$, then

$$
\Delta^{k}\left(\sum c_{n} e^{i \lambda_{n} x}\right)=\sum c_{n} O\left(n^{-\alpha k}\right) e^{i \lambda_{n} x} .
$$

Hence for $k$ sufficiently large (depending only on $\alpha$ ) the representation (2) implies that $\Delta^{k} f$ equals a smooth function a.e., so $\lambda(n)$ cannot be a Menshov spectrum for $\mathbb{R}$.

4.2. In [5] we studied Menshov spectra in the periodic case. The main results of that paper can be extended to Menshov spectra in $\mathbb{R}$. For example, Menshov spectra for $\mathbb{R}$ may be quite sparse, up to "almost Hadamarian lacunarity". More precisely:

For any $\epsilon(n)$ decreasing to zero one can construct a (symmetric) Menshov spectrum $\Lambda$ for $\mathbb{R}$ such that $\lambda(n+1) / \lambda(n)>1+\epsilon(n), n \in \mathbb{Z}^{+}$.

This is an analog of Theorem 1 from [5] and the proof is basically the same.

\section{REFERENCES}

[1] N. Bary, A Treatise on Trigonometric Series, vol. II, Pergamon Press Inc., NY (1964). MR 30:1347

[2] R.S. Davtjan, The representation of measurable functions by Fourier integrals, Akad. Nauk. Armjan. SSR Dokl. 53 (1971), 203-207. (Russian, Armenian abstract) MR 45:4058

[3] A. Olevskiǔ, Completeness in $L^{2}(\mathbf{R})$ of almost integer translates, C.R. Acad. Sci. Paris, Sèr. I Math., 324 (1997), 987-991. MR 98a:42002 
[4] G. Kozma and A. Olevskiǐ, Representations of non-periodic functions by trigonometric series with almost integer frequencies, C.R. Acad. Sci. Paris, Sèr. I Math., 329 (1999), 275-280. MR 2000e: 42001

[5] G. Kozma and A. Olevskiǐ, Menshov Representation Spectra, Journal d'Analyse Mathématique, 84 (2001), 361-393. MR 2002h:42024

The Weizmann Institute of Science, Rehovot, IsRael

E-mail address: gadykozma@hotmail.com

E-mail address: gadyk@wisdom.weizmann.ac.il

School of Mathematical Sciences, Tel Aviv University, Ramat-Aviv, Israel 69978

E-mail address: olevskii@math.tau.ac.il 